\title{
Uplink Channel Estimation for Bandlimited MC-DS-CDMA Systems Relying on Long Spreading Codes
}

\author{
Shuai Wang ${ }^{\dagger *}$, Jing-yi Lu ${ }^{\dagger}$, Jianping $\mathrm{An}^{\dagger}$, and Lajos Hanzo*, \\ $\dagger$ School of Information Science \& Electronics, Beijing Institute of Technology, Beijing, China, \\ * School of ECS, University of Southampton, SO17 1BJ, United Kingdom. \\ Tel: +44 238059 3125, Fax: +44 2380594508 \\ Email: swang@ecs.soton.ac.uk, espanastella@sina.com, an@bit.edu.cn, lh@ecs.soton.ac.uk \\ http://www-mobile.ecs.soton.ac.uk
}

\begin{abstract}
This paper considers pilot-based parameter estimation for bandlimited MC-DS-CDMA systems relying on long spreading codes. Three different schemes are proposed and compared. The two so-called unstructured algorithms, namely the Least Squares Estimator (LS-E) and the Least Absolute Shrinkage and Selection Operator Estimator (LASSOE) first estimate the composite channel impulse response, and then extract the propagation delay, amplitude and phase. By contrast, the third algorithm namely the Structured LS Search Estimator (SLSS-E) exploits the $a$ priori knowledge of the chip waveform and directly estimates the channel parameters. Parallel interference cancelation (PIC) is incorporated in the SLSS-E for the sake of mitigating the effect of multiple access interference and hence to further improve the performance. The complexity of PIC assisted SLSS-E and LS-E only increases linearly with the number of users $K$, with the number of subcarriers $U$ and with the length of the pilot sequence $N_{\mathrm{t}}$. Simulation results indicate that the PIC assisted structured estimator outperforms its unstructured counterparts.
\end{abstract}

\section{INTRODUCTION}

As a competitive candidate for the physical-layer technique in future wireless networks, Multi-Carrier Direct-Sequence Code Division Multiple Access (MC-DS-CDMA) is proposed for example for the evolution of the High Speed Package Access (HSPA) system, where the detrimental effect of the uplink Multiple Access Interference (MAI) may be mitigated by Multiple User Detection (MUD) [1]. The performance of MUD rests a lot with channel estimation. However, open literatures on channel estimation for MC-DS-CDMA systems [2-5] often rely on the assumptions of short spreading codes and/or rectangular chip waveforms. Extension of these available solutions to accommodate more practical long spreading codes and bandlimited chip waveforms is in general not straightforward. Recently Buzzi et. al. proposed a pilot-assisted parameter estimator for single-carrier (SC) long-code aided bandlimited DS-CDMA [6]. Their scheme first estimates the the Composite Channel Impulse Response (CCIR) using the Least Squares (LS) criterion, and then extracts the parameters required for MUD. In the literature, such a "two-step" approach is often termed as being unstructured [7], in contrast to the structured schemes which directly estimate the channel parameters by exploiting a priori knowledge of the chip waveform, as advocated in [8] for bandlimited short-code based SC-DS-CDMA system. More recently another unstructured strategy is proposed in [9] where the authors argues that the CCIR is usually a sparse vector, since only a few of its entries may assume non-zero values. A new CCIR estimator is introduced therein to take advantage of such sparsity based on the so-called Least Absolute Shrinkage and Selection Operator (LASSO) [10], which performs better than the LS approach [6].

Against the above-stated background, the main concern of this paper is the channel estimation for bandlimited long-code MCDS-CDMA systems. By generalizing the results of [6], we first present our formulation of such systems in Section II. Then we extend the unstructured LS and LASSO based estimators of [6, 9] to multicarrier transmission in Sections III and IV, respectively. Furthermore, a novel structured estimator is described in Section

Acknowledgments: The financial support of the China Scholarship Council (CSC) and the National High Technology Research and Development Program of China (under grant 2009AA01Z232) is gratefully acknowledged.
V. In contrast to the approach of [8] where the MAI is treated as colored Gaussian noise and suppressed via whitening techniques, the proposed structured estimator resorts to Parallel Interference Cancelation (PIC) $[11,12]$ which makes no assumptions concerning the statistics of the MAI and involves no on-line matrix inversion. Section VI is devoted to implementation issues. Specifically, we demonstrate that the complexity of PIC assisted structured estimator is linearly dependent on the number of users $K$, on the number of subcarriers $U$ and on the length of pilot sequence $N_{\mathrm{t}}$. The similar trend is also shown to be valid for the unstructured LS estimator, whose complexity has been overestimated in [6]. In Section VII we provide our performance evaluation results.

Notations: Throughout this paper, we use $\mathbb{R}^{M \times N}$ and $\mathbb{C}^{M \times N}$ to denote $(M \times N)$-element real or complex valued matrices. The $(M \times$ $M)$ identity and zero matrices are written as $\boldsymbol{I}_{M}$ and $\boldsymbol{O}_{M}$. By E(·), $(\cdot)^{\mathrm{T}},(\cdot)^{\mathrm{H}},(\cdot)^{\dagger},\|\cdot\|_{1}$, and $\|\cdot\|_{2}$ we denote the mathematic expectation, transpose, conjugate transpose, Moore-Penrose pseudo-inverse, $\ell-1$ and $\ell-2$ norm, respectively. The symbols $*$ and $\otimes$ represent linear convolution and Kronecker product.

\section{SYSTEM MODEL}

We consider the asynchronous uplink of a bandlimited long-code aided MC-DS-CDMA system that supports $K$ active users, all of whom transmit on the same $U$ subcarriers. We assume that the $U$ subchannels of each user are sufficiently far apart so that they do not overlap with each other [13]. Then the baseband equivalent of the signal received on the $u$-th subcarrier is given by:

$r^{(u)}(t)=\sum_{k=0}^{K-1} \sum_{q=-\infty}^{+\infty} A_{k} b_{k}(q) \tilde{s}_{k, q}^{(u)}\left(t-\tau_{k}-q T_{b}\right) * c_{k}^{(u)}(t)+w^{(u)}(t)$.

In the above expression, $T_{b}$ is the duration of symbol interval, and $b_{k}(q) \in(-1,+1)$ is the $q$-th bipolar information symbol transmitted by the $k$-th user. Furthermore, $A_{k}$ and $\tau_{k}$ represent the amplitude and propagation delay of the $k$-th user's signal, while $w^{(u)}(t)$ is the zero-mean complex-valued white Gaussian noise with Power Spectral Density (PSD) equal to $2 N_{0}$. Since each subband signal is assumed to experience time-invariant block fading during a packet's transmission, we may characterize the fading channel of the $k$-th user on the $u$-th subcarrier by its impulse response of $c_{k}^{(u)}(t)=\sum_{l=0}^{L-1} \bar{\alpha}_{k, l}^{(u)} \delta(t-$ $\bar{\tau}_{k, l}$ ), where $L$ is the number of resolvable paths. All the various estimators to be presented aim for estimating the parameters $\tau_{k, l}=$ $\tau_{k}+\bar{\tau}_{k, l}, a_{k, l}^{(u)}=\left|\alpha_{k, l}^{(u)}\right|=\left|A_{k} \bar{\alpha}_{k, l}^{(u)}\right|$, and $\phi_{k, l}^{(u)}=\arg \left[\alpha_{k, l}^{(u)}\right]=$ $\arg \left[\bar{\alpha}_{k, l}^{(u)}\right]$. Moreover, the user-specific signature waveform in Eq.(1) is given by $\tilde{s}_{k, q}^{(u)}(t)=\sum_{n=0}^{N-1} \beta_{k, q}^{(u)}(n) h_{\mathrm{SRRC}}\left(t-n T_{c}\right)$, where $\beta_{k, q}^{(u)}(n)$ $(n=0,1, \cdots, N-1)$ is the pseudo-noise (PN) code employed by user $k$ for spreading its $q$-th data bit on the $u$-th subcarrier, while $N$ is the Time-Domain (TD) spreading factor. Without loss of generality, we assume that the bandlimited chip pulse $h_{\mathrm{SRRC}}(t)$ is a squareroot raised-cosine waveform which is time-limited to $\left[0,4 T_{c}\right)[6,14]$. Accordingly, the chip-matched filter is $h_{\mathrm{SRRC}}\left(4 T_{c}-t\right)$. Convolving 
both sides of Eq.(1) with $h_{\mathrm{SRRC}}\left(4 T_{c}-t\right)$, we arrive at:

$r^{(u)}(t)=\sum_{k=0}^{K-1} \sum_{q=-\infty}^{+\infty} A_{k} b_{k}(q) s_{k, q}^{(u)}\left(t-\tau_{k}-q T_{b}\right) * c_{k}^{(u)}(t)+n^{(u)}(t)$

where $h_{\mathrm{RC}}(t)$ represents a raised cosine chip waveform time-limited to $\left[0,8 T_{c}\right)$ and $s_{k, q}^{(u)}(t)=\sum_{n=0}^{N-1} \beta_{k, q}^{(u)}(n) h_{\mathrm{RC}}\left(t-n T_{c}\right)$. Finally, we note that $n^{(u)}(t)=w^{(u)}(t) * h_{\mathrm{SRRC}}\left(4 T_{c}-t\right)$ is a colored Gaussian noise process.

Upon denoting the effective signature waveform by $h_{k, q}^{(u)}\left(t ; \tau_{k}\right)=$ $A_{k} s_{k, q}^{(u)}\left(t-\tau_{k}\right) * c_{k}^{(u)}(t)$, Eq.(2) can be rewritten as:

$$
r^{(u)}(t)=\sum_{k=0}^{K-1} \sum_{q=-\infty}^{+\infty} b_{k}(q) h_{k, q}^{(u)}\left(t-q T_{b}, \tau_{k}\right)+n^{(u)}(t) .
$$

Assuming $0 \leqslant\left(\tau_{k}+T_{m}\right)<T_{b}$ where $T_{m}$ stands for the maximum delay spread [6], it may be readily seen that $h_{k, q}^{(u)}\left(t-q T_{b} ; \tau_{k}\right)$ has a TD support of $\left[q T_{b},(q+2) T_{b}+7 T_{c}\right]$, hence during the $q$-th symbol interval $\mathcal{T}_{q} \triangleq\left[q T_{b},(q+1) T_{b}\right]$, the term $\sum_{q=0}^{B-1} b_{k}(q) h_{k, q}^{(u)}\left(t-q T_{b} ; \tau_{k}\right)$ in Eq.(3) is influenced by at most three consecutive bits, namely by $\left\{b_{k}(q-2), b_{k}(q-1), b_{k}(q)\right\}$. Given the sampling rate equal to $\left(M / T_{c}\right)$ per sec, the vector $\boldsymbol{r}^{(u)}(q) \in \mathbb{C}^{M N \times 1}$ that contains the $M N$ samples of $r^{(u)}(t)$ coming from $\mathcal{T}_{b}$ may be formulated as:

$$
\begin{gathered}
\boldsymbol{r}^{(u)}(q)=\sum_{k=0}^{K-1}\left[b_{k}(q-2) \boldsymbol{h}_{k, q-2}^{(u)}+b_{k}(q-1) \boldsymbol{h}_{k, q-1}^{(u)}+\right. \\
\left.b_{k}(q) \boldsymbol{h}_{k, q}^{(u)}\right]+\boldsymbol{n}^{(u)}(q),
\end{gathered}
$$

where $\boldsymbol{h}_{k, q-2}^{(u)}, \boldsymbol{h}_{k, q-1}^{(u)}, \boldsymbol{h}_{k, q}^{(u)}$, and $\boldsymbol{n}^{(u)}(q)$ comprise the $M N$ samples of $h_{k, q-2}^{(u)}\left[t-(q-2) T_{b} ; \tau_{k}\right], h_{k, q-1}^{(u)}\left[\left(t-(q-1) T_{b} ; \tau_{k}\right], h_{k, q}^{(u)}(t-\right.$ $\left.q T_{b} ; \tau_{k}\right)$, and $n^{(u)}(t)$ during $\mathcal{T}_{q}$. Here oversampling factor $M$ can be any positive integer larger than one.

Similar to the effective signature waveform of $h_{k, q}^{(u)}\left(t ; \tau_{k}\right)$, we may construct the so-called effective chip pulse:

$$
g_{k}^{(u)}\left(t ; \tau_{k}\right)=A_{k} h_{\mathrm{RC}}\left(t-\tau_{k}\right) * c_{k}^{(u)}(t) .
$$

As $0 \leqslant\left(\tau_{k}+T_{m}\right)<T_{b}, g_{k}^{(u)}\left(t ; \tau_{k}\right)$ is time-limited to $\left[0, T_{b}+8 T_{c}\right)$. By observing that we have $h_{k, q}^{(u)}\left(t ; \tau_{k}\right)=\sum_{n=0}^{N-1} \beta_{k, q}^{(u)}(n) g_{k}^{(u)}(t-$ $\left.n T_{c} ; \tau_{k}\right)$ and defining $\boldsymbol{g}_{k}^{(u)} \in \mathbb{C}^{(M N+8 M-1) \times 1}$ as:

$$
\boldsymbol{g}_{k}^{(u)}=\left\{g_{k}^{(u)}\left[\frac{T_{c}}{M} ; \tau_{k}\right], \cdots, g_{k}^{(u)}\left[T_{b}+\frac{(8 M-1) T_{c}}{M} ; \tau_{k}\right]\right\}^{\mathrm{T}},
$$

we may arrive at: $\boldsymbol{h}_{k, q-2}^{(u)}=\boldsymbol{C}_{k,-2}^{(u)}(q) \boldsymbol{g}_{k}^{(u)}, \boldsymbol{h}_{k, q-1}^{(u)}=\boldsymbol{C}_{k,-1}^{(u)}(q) \boldsymbol{g}_{k}^{(u)}$, and $\boldsymbol{h}_{k, q}^{(u)}=\boldsymbol{C}_{k, 0}^{(u)}(q) \boldsymbol{g}_{k}^{(u)}$, where $\boldsymbol{C}_{k,-2}^{(u)}(q), \boldsymbol{C}_{k,-1}^{(u)}(q)$, and $\boldsymbol{C}_{k, 0}^{(u)}(q)$ are $M N \times(M N+8 M-1)$ dimensional matrices determined by $\beta_{k, q}^{(u)}(n)^{1}$. Then the following more compact formulations of $\boldsymbol{r}^{(u)}(q)$ may be obtained:

$$
\begin{aligned}
\boldsymbol{r}^{(u)}(q) & =\sum_{k=0}^{K-1} \boldsymbol{A}_{k}^{(u)}(q) \boldsymbol{g}_{k}^{(u)}+\boldsymbol{n}^{(u)}(q) \\
& =\boldsymbol{A}^{(u)}(q) \boldsymbol{g}^{(u)}+\boldsymbol{n}^{(u)}(q),
\end{aligned}
$$

where we have $\boldsymbol{A}_{k}^{(u)}(q)=b_{k}(q-2) \boldsymbol{C}_{k,-2}^{(u)}(q)+b_{k}(q-1) \boldsymbol{C}_{k,-1}^{(u)}(q)+$ $b_{k}(q) \boldsymbol{C}_{k, 0}^{(u)}(q), \boldsymbol{A}^{(u)}(q)=\left[\boldsymbol{A}_{0}^{(u)}(q), \cdots, \boldsymbol{A}_{K-1}^{(u)}(q)\right], \boldsymbol{g}^{(u)}=$ $\left\{\left[\boldsymbol{g}_{0}^{(u)}\right]^{\mathrm{T}}, \cdots,\left[\boldsymbol{g}_{K-1}^{(u)}\right]^{\mathrm{T}}\right\}^{\mathrm{T}}$.

If the first $N_{\mathrm{t}}$ symbols sent by each user $\left\{b_{k}(q) \mid \begin{array}{l}k=0, \cdots, K-1 . \\ q=0, \cdots, N_{\mathrm{t}}-1\end{array}\right\}$ are known to the receiver as pilots, then we can stack the received data collected within $N_{\mathrm{t}}$ consecutive symbol interval on all $U$ subchannels into a $U N_{\mathrm{t}} M N$-dimensional vector $\boldsymbol{r}=\left\{\left[\boldsymbol{r}^{(1)}(0)\right]^{\mathrm{T}}, \cdots,\left[\boldsymbol{r}^{(1)}\left(N_{\mathrm{t}}-\right.\right.\right.$

${ }^{1}$ For detailed structure of $\boldsymbol{C}_{k,-2}^{(u)}(q), \boldsymbol{C}_{k,-1}^{(u)}(q)$ and $\boldsymbol{C}_{k, 0}^{(u)}(q)$, please see Eq.(9)-(11) in [6].
$\left.1)]^{\mathrm{T}}, \cdots,\left[\boldsymbol{r}^{(U)}(0)\right]^{\mathrm{T}}, \cdots,\left[\boldsymbol{r}^{(U)}\left(N_{\mathrm{t}}-1\right)\right]\right\}^{\mathrm{T}}$, which takes the form:

$$
\boldsymbol{r}=\underbrace{\operatorname{diag}\left[\boldsymbol{A}^{(1)}, \cdots, \boldsymbol{A}^{(U)}\right]}_{\boldsymbol{A}} \underbrace{\left(\begin{array}{c}
\boldsymbol{g}^{(1)} \\
\vdots \\
\boldsymbol{g}^{(U)}
\end{array}\right)}_{\boldsymbol{g}}+\underbrace{\left(\begin{array}{c}
\boldsymbol{n}^{(1)} \\
\vdots \\
\boldsymbol{n}^{(U)}
\end{array}\right)}_{\boldsymbol{n}}=\boldsymbol{A} \boldsymbol{g}+\boldsymbol{n},
$$

where $\boldsymbol{A}^{(u)}=\left\{\left[\boldsymbol{A}^{(u)}(0)\right]^{\mathrm{T}}, \cdots,\left[\boldsymbol{A}^{(U)}\left(N_{\mathrm{t}}-1\right)\right]^{\mathrm{T}}\right\}^{\mathrm{T}}, \boldsymbol{n}^{(u)}=$ $\left\{\left[\boldsymbol{n}^{(u)}(0)\right]^{\mathrm{T}}, \cdots,\left[\boldsymbol{n}^{(U)}\left(N_{\mathrm{t}}-1\right)\right]^{\mathrm{T}}\right\}^{\mathrm{T}}$. In some cases we might prefer to decompose $r$ into a sum of components corresponding to different users, like:

$$
\boldsymbol{r}=\sum_{k=0}^{K-1} \underbrace{\operatorname{diag}\left[\boldsymbol{A}_{k}^{(1)}, \cdots, \boldsymbol{A}_{k}^{(U)}\right]}_{\boldsymbol{A}_{k}} \underbrace{\left(\begin{array}{c}
\boldsymbol{g}_{k}^{(1)} \\
\vdots \\
\boldsymbol{g}_{k}^{(U)}
\end{array}\right)}_{\boldsymbol{g}_{k}}=\sum_{k=0}^{K-1} \boldsymbol{A}_{k} \boldsymbol{g}_{k}+\boldsymbol{n},
$$

where $\boldsymbol{A}_{k}^{(u)}=\left\{\left[\boldsymbol{A}_{k}^{(u)}(0)\right]^{\mathrm{T}}, \cdots,\left[\boldsymbol{A}_{k}^{(u)}\left(N_{\mathrm{t}}-1\right)\right]^{\mathrm{T}}\right\}^{\mathrm{T}}$.

To handle the colored noise vector $\boldsymbol{n}$ that may affect the estimation quality, we can multiply both sides of Eq.(8) and Eq.(9) with a "whitening matrix" $\boldsymbol{W} \in \mathbb{R}^{U N_{\mathrm{t}} M N \times U N_{\mathrm{t}} M N}$, which leads to:

$$
\boldsymbol{y}=\boldsymbol{F} \boldsymbol{g}+\boldsymbol{w}=\sum_{k=0}^{K-1} \boldsymbol{F}_{k} \boldsymbol{g}_{k}+\boldsymbol{w}
$$

where $\boldsymbol{y}=\boldsymbol{W} \boldsymbol{r}, \boldsymbol{F}=\boldsymbol{W} \boldsymbol{A}, \boldsymbol{F}_{k}=\boldsymbol{W} \boldsymbol{A}_{k}$, and $\boldsymbol{w}=\boldsymbol{W} \boldsymbol{n}$ is a complex-valued white Gaussian vector with a zero mean and a covariance matrix of $\sigma^{2} \boldsymbol{I}_{U N_{\mathrm{t}} M N}$. If $\boldsymbol{L}$ is the Cholesky factor of the covariance matrix of $\boldsymbol{n}$, then we have $\sigma^{2}=1$ provided that $\boldsymbol{W}=\boldsymbol{L}^{-1}$ [9].

\section{LS BASEd ChANNEL Estimation (LS-E)}

Given Eq.(10), the overall CCIR $\boldsymbol{g}$ may be directly estimated by invoking the LS estimation procedure of [6]:

$$
\hat{\boldsymbol{g}}^{\mathrm{LS}}=\arg \min _{\boldsymbol{x}}\|\boldsymbol{y}-\boldsymbol{F} \boldsymbol{x}\|_{2}^{2}=\boldsymbol{F}^{\dagger} \boldsymbol{y}=\left(\boldsymbol{F}^{\mathrm{H}} \boldsymbol{F}\right)^{-1} \boldsymbol{F}^{\mathrm{H}} \boldsymbol{y} .
$$

Upon rearranging the entries in $\hat{\boldsymbol{g}}^{\mathrm{LS}}$ according to the relationships specified in Eq.(7)-(9), we can formulate $\hat{\boldsymbol{g}}_{k}^{\mathrm{LS}}$ as the estimate of the $k$ th user's CCIR $\boldsymbol{g}_{k}$. The next step is to extract the channel parameters $\left\{\tau_{k, l}, a_{k, l}^{(u)}, \phi_{k, l}^{(u)} \mid \begin{array}{l}l=1, \cdots, L \\ u=1, \cdots, U\end{array}\right\}$ from $\hat{\boldsymbol{g}}_{k}^{\mathrm{LS}}$. An in-depth description of the extraction algorithm can be found in [6] for a single carrier system and its extension to multicarrier transmission is quite natural. For conciseness, we leave the details for readers.

\section{LASSO BASED ChANNEL ESTIMATION (LASSO-E)}

The question as to why $\boldsymbol{g}$ is a sparse vector has been answered for DS-CDMA in [9], and most of the arguments hold for MC-DSCDMA as well. One way to exploit the sparsity of $\boldsymbol{g}$ is to estimate $g$ as the solution to the following LASSO problem $[9,10]$ :

$$
\hat{\boldsymbol{g}}^{\text {LASSO }}=\arg \min _{\boldsymbol{x}}\left\{\|\boldsymbol{y}-\boldsymbol{F} \boldsymbol{x}\|_{2}^{2}+\lambda|\boldsymbol{x}|_{1}\right\} .
$$

where $\lambda>0$ and the larger $\lambda$ is, the more entries of $\hat{\boldsymbol{g}}^{\text {LASSO }}$ will become zero. In [9] $\lambda$ was determined empirically in the form of $\lambda_{\mathrm{e}}=\sqrt{2 \sigma \ln [K U(M N+8 M-1)]}$. Once $\hat{\boldsymbol{g}}^{\text {LASSO }}$ was obtained, the channel parameters of each user can be extracted in the same way as for the LS-E of Section III.

\section{PIC-SLSS BASED CHANNEL ESTIMATION}

Compared to the unstructured estimators of $[6,9]$ which rely on the explicit estimation of the CCIR, structured estimators directly estimate the channel parameters and generally lead to an improved performance, since the number of unknowns to be estimated is substantially reduced [7]. To facilitate description, let us now commence our discussion of structured estimator designed for bandlimited longcode based MC-DS-CDMA with the simplest single-path single-user scenario, and then extend it step-by-step to more relevant scenarios, where both multipath interference and MAI are present. 


\section{A. Single-path Single-user Channel: the Simplest Scenario}

Without loss of generality, we assume the uplink channel is solely occupied by user $k$. Then Eq.(10) reduces to:

$$
\boldsymbol{y}=\boldsymbol{F}_{k} \boldsymbol{g}_{k}+\boldsymbol{w} .
$$

Recalling the single-path assumption of $L=1$ and invoking Eq.(5) and (6), we have: $\boldsymbol{g}_{k}^{(u)}=\alpha_{k, 1}^{(u)} \boldsymbol{h}_{\mathrm{RC}}\left(\tau_{k, 1}\right)$, where $\boldsymbol{h}_{\mathrm{RC}}\left(\tau_{k, 1}\right)=$ $\left[h_{\mathrm{RC}}\left(\frac{T_{c}}{M}-\tau_{k, 1}\right), \cdots, h_{\mathrm{RC}}\left(T_{b}+\frac{(8 M-1) T_{c}}{M}-\tau_{k, 1}\right)\right]^{\mathrm{T}}$. Hence $\boldsymbol{g}_{k}$ in Eq.(13) may be rewritten as:

$$
\boldsymbol{g}_{k}=\boldsymbol{G}\left(\tau_{k, 1}\right) \boldsymbol{\alpha}_{k, 1}
$$

where $\boldsymbol{\alpha}_{k, 1}=\left[\alpha_{k, 1}^{(1)}, \cdots, \alpha_{k, 1}^{(U)}\right]^{\mathrm{T}}$ is a $(U \times 1)$-element vector that contains the complex-valued fading coefficients of $U$ subcarriers, and the matrix $\boldsymbol{G}\left(\tau_{k, 1}\right) \in \mathbb{R}^{U(M N+8 M-1) \times U}$ is defined as $\boldsymbol{G}\left(\tau_{k, 1}\right)=$ $\boldsymbol{I}_{U} \otimes \boldsymbol{h}_{\mathrm{RC}}\left(\tau_{k, 1}\right)$. Here we use the notations $\boldsymbol{G}\left(\tau_{k, 1}\right)$ and $\boldsymbol{h}_{\mathrm{RC}}\left(\tau_{k, 1}\right)$ to emphasize the fact that both $\boldsymbol{G}$ and $\boldsymbol{h}_{\mathrm{RC}}$ are functions of $\tau_{k, 1}$. Upon substituting Eq.(14) into Eq.(13) and letting $\boldsymbol{F}_{k} \boldsymbol{G}\left(\tau_{k, 1}\right)=$ $\boldsymbol{S}_{k}\left(\tau_{k, 1}\right) \in \mathbb{R}^{U N_{\mathrm{t}} M N \times U}$, we arrive at:

$$
\boldsymbol{y}=\boldsymbol{S}_{k}\left(\tau_{k, 1}\right) \boldsymbol{\alpha}_{k, 1}+\boldsymbol{w} .
$$

From Eq.(15) we may obtain the Equivalent Log-Likelihood (ELL) function as $\mathcal{F}_{\mathrm{ELL}}\left(\boldsymbol{y} \mid \tau_{k, 1}, \boldsymbol{\alpha}_{k, 1}\right)=-\left\|\boldsymbol{y}-\boldsymbol{S}_{k}\left(\tau_{k, 1}\right) \boldsymbol{\alpha}_{k, 1}\right\|_{2}^{2}$, which implies that the joint Maximum Likelihood Estimation (MLE) of $\tau_{k, 1}$ and $\boldsymbol{\alpha}_{k, 1}$ may be formulated as:

$$
\left\{\hat{\tau}_{k, 1}, \hat{\boldsymbol{\alpha}}_{k, 1}\right\}_{\mathrm{ML}}=\underset{\left\{\tau_{k, 1}, \boldsymbol{\alpha}_{k, 1}\right\}}{\arg \min }\left\|\boldsymbol{y}-\boldsymbol{S}_{k}\left(\tau_{k, 1}\right) \boldsymbol{\alpha}_{k, 1}\right\|_{2}^{2} .
$$

To compute the joint MLE by directly solving Eq.(16) is rather challenging. However, if $\tau_{k, 1}$ is known a priori, the optimal estimate of $\boldsymbol{\alpha}_{k, 1}$ which satisfies Eq.(16) may be formulated as:

$$
\hat{\boldsymbol{\alpha}}_{k, 1}^{\mathrm{SLS}}\left(\tau_{k, 1}\right)=\boldsymbol{S}_{k}^{\dagger}\left(\tau_{k, 1}\right) \boldsymbol{y}=\left[\boldsymbol{S}_{k}^{\mathrm{H}}\left(\tau_{k, 1}\right) \boldsymbol{S}_{k}\left(\tau_{k, 1}\right)\right]^{-1} \boldsymbol{S}_{k}^{\mathrm{H}}\left(\tau_{k, 1}\right) \boldsymbol{y},
$$

where the superscript "SLS" is the abbreviation of Structured LS.

Based on Eq.(17), a grid search scheme may be developed for numerically solving Eq.(16). More particularly, we may hypothesize a specific value of $\hat{\tau}_{k, 1} \in\left[0, T_{\mathrm{b}}\right)$ and obtain the corresponding $\hat{\boldsymbol{\alpha}}_{k, 1}^{\text {SLS }}\left(\hat{\tau}_{k, 1}\right)$ from Eq.(17). Once this procedure has been repeated for all possible candidates of $\hat{\tau}_{k, 1}$, an approximate joint MLE can be found as the combination of $\left\{\tau_{k, 1}, \hat{\boldsymbol{\alpha}}_{k, 1}^{\text {SLS }}\left(\hat{\tau}_{k, 1}\right)\right\}$ which minimizes the Cost Function (CF) specified in Eq.(16). In our forthcoming discourse, we will refer to this algorithm as the Structured Least Squares Search Estimator (SLSS-E). Note that the above mentioned grid search performed by the SLSS-E is one- dimensional, proceeding across the limited range of $\hat{\tau}_{k, 1} \in\left[0, T_{\mathrm{b}}\right]$ only. As the resolution of the grid search increases, SLSS-E asymptotically converges to the MLE. Given the estimated complex channel gain $\hat{\boldsymbol{\alpha}}_{k, 1}^{\mathrm{SLS}}$, the further extraction of the amplitude and phase estimates becomes trivial.

\section{B. Multipath Single-user Scenario}

To accommodate the multipath effect, Eq.(15) should be extended as:

$$
\boldsymbol{y}=\sum_{l=1}^{L} \boldsymbol{S}_{k}\left(\tau_{k, l}\right) \boldsymbol{\alpha}_{k, l}+\boldsymbol{w} .
$$

The direct adaptation of the SLSS-E to dispersive channel entails a multi-dimensional grid search and leads to a complexity exponentially dependent on $L$. Following the approach proposed in [8], we may first apply the single-path SLSS-E to Eq.(18) and find the SLSS estimates $\left\{\hat{\tau}_{k, 1}^{\text {SLSS }}, \hat{\boldsymbol{\alpha}}_{k, 1}^{\text {SLSS }}\right\}$ corresponding to path 1, i.e., to the strongest path. Then the contribution of path 1 to the single-user multipath signal can be regenerated by $\boldsymbol{S}_{k}\left(\hat{\tau}_{k, 1}^{\text {SLSS }}\right) \hat{\boldsymbol{\alpha}}_{k, 1}^{\text {SLSS }}$ and subtracted from $\boldsymbol{y}$ of Eq.(18). Applying the single-path SLSS-E to the resultant residual, we may obtain the SLSS estimation corresponding to the 2nd-strongest path. Obviously, the entire estimation task can be completed after $L$ cycles, and this successive grid search imposes a modest complexity, which increases linearly with $L$.

\section{Multiuser Multipath Channel: the Practical Scenario}

In an uplink multiuser system, the accuracy of channel estimation is often affected by the MAI. To overcome this difficulty, we now generalize the above-mentioned SLSS-E by combining it with Parallel Interference Cancelation (PIC) $[11,12]$. This potent combination results in a new iterative estimator, which we refer to as PIC assisted SLSS-E (PIC-SLSS-E). Given the multipath, multiuser signal of:

$$
\boldsymbol{y}=\sum_{k=1}^{K} \sum_{l=1}^{L} \boldsymbol{S}_{k}\left(\tau_{k, l}\right) \boldsymbol{\alpha}_{k, l}+\boldsymbol{w}
$$

the PIC-SLSS-E scheme operates as follows:

Step 1: (Initialization) Set $v=1$, where $v$ is the index of the PIC stage. For the $k$-th user, the parameter estimate of Stage-1 $\left\{\hat{\tau}_{k, l}^{v=1}, \hat{\boldsymbol{\alpha}}_{k, l}^{v=1} \mid l=1, \cdots, L\right\}$ is obtained by applying the corresponding multipath SLSS-E to $y$ of Eq.(19). The initial value of the CF should be set to $\mathcal{C}^{v-1}=\mathcal{C}^{0}=+\infty$.

Step 2: ( Reconstruction) Based on the channel estimates obtained in the previous stage (Stage-v), the $k$-th user's contribution to the received signal may be reconstructed by:

$$
\hat{\boldsymbol{y}}_{k}^{v}=\sum_{l=1}^{L} \boldsymbol{S}_{k}\left(\hat{\tau}_{k, l}^{v}\right) \hat{\boldsymbol{\alpha}}_{k, l}^{v} .
$$

Step 3: (Verification) Calculate the CF value for the previous stage: $\mathcal{C}^{v}=\left\|\boldsymbol{y}-\sum_{k=1}^{K} \hat{\boldsymbol{y}}_{k}^{v}\right\|_{2}^{2}$, and check the following two conditions: I. $\mathcal{C}^{v}<\mathcal{C}^{v-1}$ (This is always true for $v=1$ ). II. the maximum iteration limit, say $V$, has not been reached, i.e., we still have $v \leqslant V$. Proceed to Step 4, if both of the above two conditions are satisfied. Otherwise, the PIC-SLSS-E is terminated at Step 3.

Step 4: (Subtraction) For the $k$-th user $(k=1, \cdots, K)$, the refined received signal $\boldsymbol{y}_{k}^{v+1}$ to be used for channel estimation in the $(v+1)$ - th stage is constructed as:

$$
\boldsymbol{y}_{k}^{v+1}=\boldsymbol{y}-\sum_{k^{\prime}=1, k^{\prime} \neq k}^{K} \hat{\boldsymbol{y}}_{k^{\prime}}^{v}=\boldsymbol{y}-\sum_{k^{\prime}=1}^{K} \hat{\boldsymbol{y}}_{k^{\prime}}^{v}+\hat{\boldsymbol{y}}_{k}^{v} .
$$

Step 5: (Estimation $)$ For the $k$-th user $(k=1, \cdots, K)$, the channel parameter estimates $\left\{\hat{\tau}_{k, l}^{v+1}, \hat{\boldsymbol{\alpha}}_{k, l}^{v+1} \mid l=1, \cdots, L\right\}$ generated during the $(v+1)$ - th stage are obtained by applying the multipath SLSS-E to $\boldsymbol{y}_{k}^{v+1}$. Then we set $v=v+1$ and return to Step 2 .

\section{IMPLEMENTATION ISSUES AND COMPLEXITY ANALYSIS}

\section{A. Whitening}

With the covariance matrix of the colored noise vector $\boldsymbol{n}$ in Eq.(8) denoted by $\boldsymbol{\Psi}=\mathrm{E}\left(\boldsymbol{n} \boldsymbol{n}^{H}\right)$, the whitening matrix $\boldsymbol{W}$ has to satisfy $\boldsymbol{W} \boldsymbol{\Psi} \boldsymbol{W}^{\mathrm{H}}=\sigma^{2} \boldsymbol{I}_{U N_{\mathrm{t}} M N}$, where $\sigma^{2}$ is the variance of the whitened noise. As $\sigma^{2}$ may assume arbitrary positive values, $\boldsymbol{W}$ is not unique. Recalling $\boldsymbol{n}=\left\{\left[\boldsymbol{n}^{(1)}\right]^{\mathrm{T}}, \cdots,\left[\boldsymbol{n}^{(U)}\right]^{\mathrm{T}}\right\}^{\mathrm{T}}$ from Eq.(8), and observing the noise vectors in the $U$ different subchannels are independent of each other, then for any $u_{1}$ and $u_{2} \in\{1, \cdots, U\}$ we have:

$$
\mathrm{E}\left\{\boldsymbol{n}^{\left(u_{1}\right)}\left[\boldsymbol{n}^{\left(u_{2}\right)}\right]^{\mathrm{H}}\right\}=\left\{\begin{array}{cc}
\boldsymbol{\Phi}, & u_{1}=u_{2}, \\
\boldsymbol{O}_{N_{\mathrm{t}} M N}, & u_{1} \neq u_{2},
\end{array}\right.
$$

where the $(i, j)$-th entry of $\boldsymbol{\Phi} \in \mathbb{R}^{N_{\mathrm{t}} M N \times N_{\mathrm{t}} M N}$ equals to $2 N_{0} h_{\mathrm{RC}}\left(4 T_{\mathrm{c}}+|i-j| T_{\mathrm{c}} / M\right)$ [6]. Upon setting $N_{0}=1$ and obtaining the corresponding whitening matrix for $\boldsymbol{\Phi}$, say $\boldsymbol{P} \in$ $\mathbb{R}^{N_{\mathrm{t}} M N \times N_{\mathrm{t}} M N}$, we may readily verify that $\widetilde{\boldsymbol{W}}=\operatorname{diag}[\boldsymbol{P}, \cdots, \boldsymbol{P}] \in$ $\mathbb{R}^{U N_{\mathrm{t}} M N \times U N_{\mathrm{t}} M N}$ is a whitening matrix for $\Psi$.

The above discussions suggest that the on-line calculation of the whitening matrix may be avoided by pre-computing the real-valued matrix $\boldsymbol{P}$ and storing it in the memory. The resultant memory requirements and the computational complexity associated with $y=\widetilde{\boldsymbol{W}} \boldsymbol{r}$ have been summarized in Tab I. 
TABLE I

SUMMARY OF COMPLEXITY ANALYSIS

\begin{tabular}{|l||c|c|c|}
\hline & Real Multiplication & Real Addition & Memory Expenditure \\
\hline \hline Whitening & $2 U\left(N_{\mathrm{t}} M N\right)^{2}$ & $2 U\left(N_{\mathrm{t}}^{2} M^{2} N^{2}-N_{\mathrm{t}} M N\right)$ & $N_{\mathrm{t}}^{2} M^{2} N^{2}$ \\
\hline LS-E & $2 U N_{\mathrm{t}}\left(M^{2} N^{2}+8 M^{2} N-M N\right) K$ & $2 U\left(N_{\mathrm{t}} M N-1\right)(M N+8 M-1) K$ & $U N_{\mathrm{t}}\left(M^{2} N^{2}+8 M^{2} N-M N^{2} K\right.$ \\
\hline PIC-SLSS-E & $2 U N_{\mathrm{t}} M N[K L(3 N R+1)+1] V$ & $\begin{array}{c}6\left(U N_{\mathrm{t}} M N^{2}-2 U N-N\right) R K L V \\
+2 U N_{\mathrm{t}} M N[K(L+2)+1] V-V\end{array}$ & $2 R K U N_{\mathrm{t}} M N^{2}$ \\
\hline
\end{tabular}

$\star$ The negligible overhead of parameter extraction in LS-E has been omitted.

\section{B. $L S-E$}

Following the arguments of [6], the complexity of the LS-E is $\mathcal{O}\left[K^{3} U^{3}(M N+8 M-1)^{3}\right]$ due to the inversion of $\left(\boldsymbol{F}^{\mathrm{H}} \boldsymbol{F}\right)$ in Eq.(11), or at least $\mathcal{O}\left[K^{2} U^{2}(M N+8 M-1)^{2}\right]$ by Gauss-Seidel iterations. However, this is actually not so much of a problem since $\boldsymbol{F}^{\dagger}=\left(\boldsymbol{F}^{\mathrm{H}} \boldsymbol{F}\right)^{-1} \boldsymbol{F}^{\mathrm{H}}$ can be computed off-line. To see this, recall from Eq.(10) that we have $\boldsymbol{F}=\boldsymbol{W} \boldsymbol{A}$, where $\boldsymbol{W}$ can be pre-computed as $\widetilde{\boldsymbol{W}}$, and $\boldsymbol{A}$ is solely dependent on the spreading codes and the pilot symbols, both of which are known to the receiver. Therefore, the implementational cost associated with the LS-E of Eq.(11) is contributed by the memory space required for storing $\boldsymbol{F}^{\dagger}$ and the computational complexity of calculating $\boldsymbol{F}^{\dagger} \boldsymbol{y}$. Moreover, a close inspection of $\boldsymbol{F}$ reveals that $\boldsymbol{F}^{\dagger}$ obeys the structure of $\boldsymbol{F}^{\dagger}=\operatorname{diag}\left\{\left[\boldsymbol{F}^{(1)}\right]^{\dagger}, \cdots,\left[\boldsymbol{F}^{(U)}\right]^{\dagger}\right\}$, where $\left[\boldsymbol{F}^{(u)}\right]^{\dagger}=$ $\left\{\left[\boldsymbol{P} \boldsymbol{A}^{(u)}\right]^{\mathrm{H}}\left[\boldsymbol{P} \boldsymbol{A}^{(u)}\right]\right\}^{-1}\left[\boldsymbol{P} \boldsymbol{A}^{(u)}\right]^{\mathrm{H}}$, provided that $\widetilde{\boldsymbol{W}}$ is used as the whitening matrix. Detailed complexity summary of the LS-E can be found in Tab I.

\section{PIC-SLSS-E}

The SLSS-E given in Eq.(17) may also exploit pre-computation to avoid on-line matrix inversion. Moreover, the complexity imposed can be further reduced if we exploit the inherent structure of the matrix $\boldsymbol{S}_{k}\left(\tau_{k}\right)$ and $\boldsymbol{S}_{k}^{\dagger}\left(\tau_{k}\right)$ in Eq.(15)-Eq.(17): ${ }^{2}$

$$
\begin{aligned}
& \boldsymbol{S}_{k}\left(\tau_{k}\right)=\operatorname{diag}\left[\boldsymbol{S}_{k}^{(1)}\left(\tau_{k}\right), \cdots, \boldsymbol{S}_{k}^{(U)}\left(\tau_{k}\right)\right], \\
& \boldsymbol{S}_{k}^{\dagger}\left(\tau_{k}\right)=\operatorname{diag}\left\{\left[\boldsymbol{S}_{k}^{(1)}\left(\tau_{k}\right)\right]^{\dagger}, \cdots,\left[\boldsymbol{S}_{k}^{(U)}\left(\tau_{k}\right)\right]^{\dagger}\right\},
\end{aligned}
$$

where $\boldsymbol{S}_{k}^{(u)}\left(\tau_{k}\right)=\boldsymbol{P} \boldsymbol{A}_{k}^{(u)} \boldsymbol{h}_{\mathrm{RC}}\left(\tau_{k}\right)$, and $\left[\boldsymbol{S}_{k}^{(u)}\left(\tau_{k}\right)\right]^{\dagger}=$ $\left\{\left[\boldsymbol{S}_{k}^{(u)}\left(\tau_{k}\right)\right]^{\mathrm{H}} \boldsymbol{s}_{k}^{(u)}\left(\tau_{k}\right)\right\}^{-1}\left[\boldsymbol{s}_{k}^{(u)}\left(\tau_{k}\right)\right]^{\mathrm{H}}$. Assuming that $R$ equallyspaced hypotheses are considered within every chip duration $T_{\mathrm{c}}$, the total memory requirements of storing $\boldsymbol{s}_{k}^{(u)}\left(\tau_{k}\right)$ and $\left[\boldsymbol{s}_{k}^{(u)}\left(\tau_{k}\right)\right]^{\dagger}$ for all the $K$ users are listed in Tab I.

Let us now consider the computational complexity of PIC-SLSSE, commencing with the Step $\mathbf{5}$ of Section V-C, and assuming that the estimation of $\left\{\tau_{k, 1}, \boldsymbol{\alpha}_{k, 1}\right\}$ in stage $v^{\prime}$ is now underway ${ }^{3}$. After testing all the $R N$ hypotheses involved in the grid search, we obtain the parameter estimates $\left\{\hat{\tau}_{k, 1}, \hat{\boldsymbol{\alpha}}_{k, 1}\right\}$ corresponding to the 1 st path of user $k$. Then the 1 st path's contribution to user $k$ 's signal $\boldsymbol{y}_{k}^{v^{\prime}}$ may be reconstructed as $\hat{\boldsymbol{y}}_{k, 1}^{v^{\prime}}=\boldsymbol{s}_{k}\left(\hat{\tau}_{k, 1}\right) \hat{\boldsymbol{\alpha}}_{k, 1}$. Upon subtracting $\hat{\boldsymbol{y}}_{k, 1}^{v^{\prime}}$ from $\boldsymbol{y}_{k}^{v^{\prime}}$, these steps may be repeated for estimating the parameters associated with the 2 nd path of user $k$. As a by-product, we obtain the residual of $\boldsymbol{y}_{k}^{v^{\prime}}$ after all the $L$ paths of user $k$ were identified, which is given by $\tilde{\boldsymbol{y}}_{k}^{v^{\prime}}=\boldsymbol{y}_{k}^{v^{\prime}}-\sum_{l=1}^{L}\left(\hat{\boldsymbol{y}}_{k, l}^{v^{\prime}}\right)$. The computations involved in the above mentioned procedure are summarized below for $K$ active users and $L$ paths per user, where both the amount of Real-valued Multiplications (RM) and Additions (RA) are provided:

$\diamond$ Grid search:

RM: $6 U N_{\mathrm{t}} M N^{2} R K L$; RA: $\left(6 U N_{\mathrm{t}} M N^{2}-2 U N-N\right) R K L$.

$\diamond$ Reconstructing all path components: RM: $2 U N_{\mathrm{t}} M N K L$.

$\diamond$ Subtracting all path components: RA: $2 U N_{\mathrm{t}} M N K L$.

The next step that follows Step 5 is Step 2 (see Section V-C). Upon exploiting the by-product $\tilde{\boldsymbol{y}}_{k}^{v^{\prime}}$ generated during Step $\mathbf{5}$ as

${ }^{2}$ Subscripts representing the multipath components have been dropped for notation convenience.

${ }^{3}$ We note that Step 5 is described for stage $(v+1)$ in Section V-C. Hence here $v^{\prime}$ can be interpreted as $v^{\prime}=v+1$. mentioned, we may introduce a low-complexity alternative to Eq.(20) for reconstruction, namely:

$$
\hat{\boldsymbol{y}}_{k}^{v^{\prime}}=\boldsymbol{y}_{k}^{v^{\prime}}-\tilde{\boldsymbol{y}}_{k}^{v^{\prime}}
$$

where $2 U N_{\mathrm{t}} M N K$ RAs are required for reconstructing all the $K$ users' signals. As for Step $\mathbf{3}$, the calculation of $\mathcal{C}^{v^{\gamma}}=\| \boldsymbol{y}-$ $\sum_{k=1}^{K} \hat{\boldsymbol{y}}_{k}^{v^{\prime}} \|_{2}^{2}$ involves $2 U N_{\mathrm{t}} M N$ RMs and $2 U N_{\mathrm{t}} M N(K+1)-1$ RAs. The last constituent part of the PIC stage is Step 4, where we evaluate $\boldsymbol{y}_{k}^{v^{\prime}+1}=\boldsymbol{y}-\left(\sum_{k^{\prime}=1}^{K} \hat{\boldsymbol{y}}_{k^{\prime}}^{v^{\prime}}\right)+\hat{\boldsymbol{y}}_{k}^{v^{\prime}}$ for each user. Note that $\left(\boldsymbol{y}-\sum_{k^{\prime}=1}^{K} \hat{\boldsymbol{y}}_{k^{\prime}}^{v^{\prime}}\right)$ has already been calculated during Step 3, hence Step 4 only entails $2 U N_{\mathrm{t}} M N K$ RAs. Assuming the PIC runs for $V$ stages, we report the complexity of PIC-SLSS-E also in Tab I.

\section{LASSO-E}

Iterative approaches $[10,15]$ have been proposed for solving the LASSO based channel estimation problem formulated by Eq.(12). However, to the best of our knowledge, there is no closed-form LASSO solution available, which makes the evaluation of its complexity quite a challenge. Owing to the lack of a closed-form solution, LASSO-E cannot pre-calculate any quantitative for the sake of saving on-line computational complexity, which constitutes an obvious disadvantage in contrast to LS-E and PIC-SLSS-E. Moreover, the whitened noise variance $\sigma^{2}$ must be estimated in advance to determine $\lambda_{\mathrm{e}}$. In the simulations, we assume $\sigma^{2}$ to be known and solve Eq.(12) as a quadratic programming problem with the so-called SeDuMi toolbox [16] aided with a Yalmip interface [17].

\section{NUMERICAL EXAMPLES}

The key system parameters adopted in our simulations are $K=5$, $N=16, M=2, N_{\mathrm{t}}=10$ and $U=4$. The long spreading codes are generated as random bipolar sequences where every entry has the same probability of assuming +1 or -1 . As in [6], we consider a near-far ratio (NFR) of $10 \mathrm{~dB}$, where the power of active users are randomly fluctuated around their average with $\pm 5 \mathrm{~dB}$ deviation, and we report the simulation results of the weakest user to characterize the lower bound of the uplink channel estimation quality. The parameter extraction in LS-E and LASSO-E is performed at a resolution of $T_{\mathrm{c}} / 10$, while we set $R=10$ for PIC-SLSS-E for the sake of fair comparison. Unless otherwise stated, $V$ is set to 4 , i.e., the PIC-SLSSE iterates for at most three stages after initialization. All results were averaged over 1000 independent runs.

In Fig. 1 we compare the probability of correct acquisition $\left(P_{\mathrm{ca}}\right)$ of the three proposed estimators for transmission both over singleand multi-path channel. The acquisition of a certain path is deemed as correct if the corresponding delay estimation error has an absolute value less than $T_{c} / 2$. We observe that the $P_{\text {ca }}$ of the SLSS-E without employing PIC (hence referred to as "unassisted") fails to approach $100 \%$ even when $E_{\mathrm{b}} / N_{0}$ is quite high. This is because the unassisted SLSS-E scheme acts essentially in a decentralized way, i.e., the MAI is not mitigated. Furthermore, although a notable performance discrepancy is observed between $V=1$ and $V=2$, increasing $V$ brings little gain when $V \geq 4$. As expected, LASSO-E is superior to LS-E, but both of them are outperformed by PIC-SLSS-E for $V \geq 2$.

In order to evaluate the channel parameter estimation quality, in Fig.2-4 we illustrate the unconditioned Mean Squared Error (MSE) and MSE conditioned on correct acquisition (ca). As for delay estimation, there is a large disparity between the conditioned and 

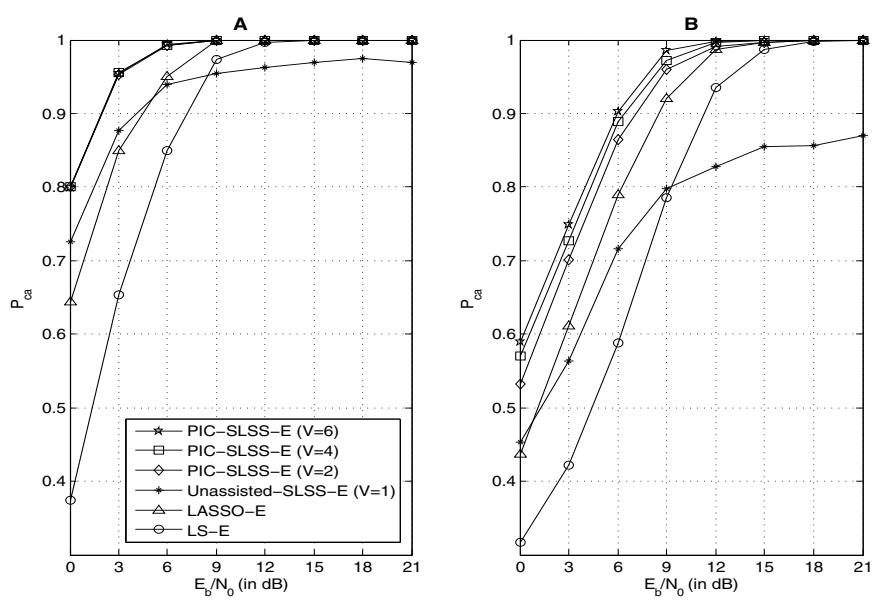

Fig. 1. The probability of correct acquisition v.s. $E_{b} / N_{0}$. NFR $=10 \mathrm{~dB}$. A $L=1$. B: $L=3$.
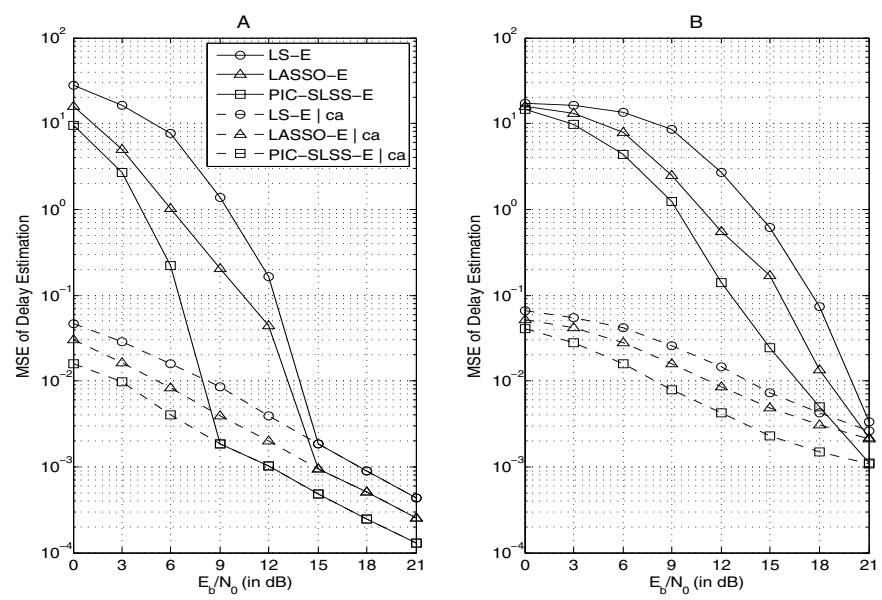

Fig. 2. The MSE of delay estimation (normalized by $T_{\mathrm{c}}^{2}$ ) v.s. $E_{b} / N_{0}$. NFR $=10 \mathrm{~dB} . \mathbf{A}: L=1$. B: $L=3$.

unconditioned MSE in the low $E_{\mathrm{b}} / N_{0}$ range, but they tend to coincide with each other in the high $E_{\mathrm{b}} / N_{0}$ range, as $P_{\mathrm{ca}}$ tends to unity. Interestingly, the difference between the conditioned and unconditioned MSE is less evident in amplitude and phase offset estimation. In general, these results demonstrate again that the structured PICSLSS-E compares favorably with the two unstructured algorithms.

\section{REFERENCES}

[1] L. Hanzo, L.-L. Yang, E. L. Kuan, and K. Yen, Single- and Multi-Carrier DS-CDMA: Multi-User Detection, Space-Time Spreading, Synchronisation, Standards and Networking, John Wiley \& Sons. ISBN 0-470-863099, 2003.

[2] J. Namgoong, T. F. Wong, and J. S. Lehnert, "Subspace multiuser detection for multicarrier DS-CDMA," in IEEE Trans. on Commun. vol.48, no.11, pp. 1897-1908, Nov. 2000.

[3] S. Buzzi, E. Grossi, and M. Lops, "Timing-free blind multiuser detection for multicarrier DS/CDMA systems with multiple antennae," in Eurosip Journal on Applied Signal Processing, no.5, pp.613-628, May 2004.

[4] D. J. Sadler, and A. Manikas, "MMSE multiuser detection for array multicarrier DS-CDMA in fading channels," in IEEE Trans. Signal Processing, vol.53, no.7, pp. 2348-2358, July 2005.

[5] B. Hu, L.-L. Yang, and L. Hanzo, "Time- and frequency-domainspread generalized multicarrier DS-CDMA using subspace-based blind and group-blind space-time multiuser detection," in IEEE Trans. Vehicular Tech., vol.57, no.5, pp.3235-3241, Sept. 2008.

[6] S. Buzzi, and V. Massaro, "Parameter estimation and multiuser detection for bandlimited long-code CDMA systems," in IEEE Trans. on Wireless Commun., vol.7, no.6, pp. 2307-2317, Jun. 2008. vol.1, pp.447-454.

[7] G. Caire, and U. Mitra, "Structured multiuser channel estimation for block-synchronous DS/CDMA," in IEEE Trans. on Commun., vol.49, no.9, pp. 1605-1617, Sept. 2001.
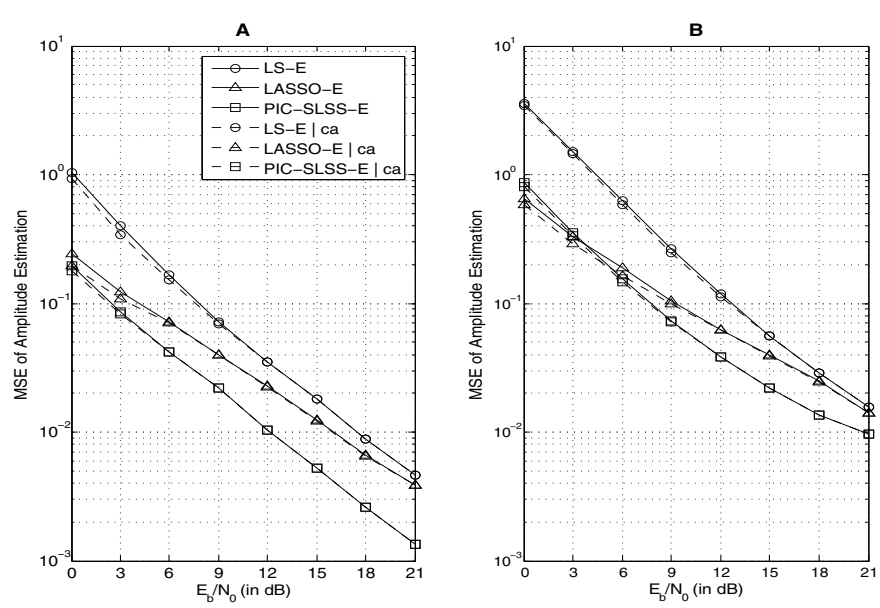

Fig. 3. The MSE of amplitude estimation (normalized by the mean value of the true squared amplitude) v.s. $E_{b} / N_{0} . \mathrm{NFR}=10 \mathrm{~dB}$. A: $L=1$. B: $L=3$.
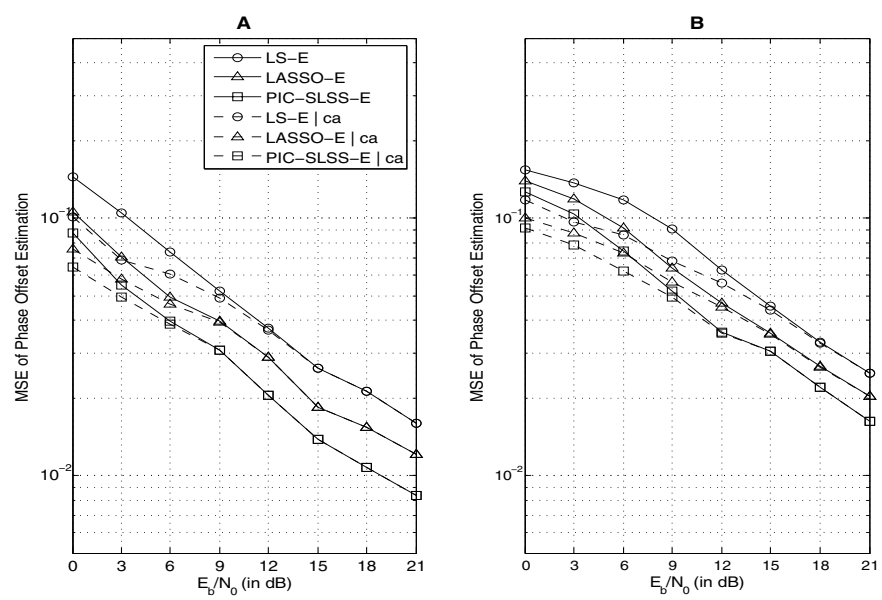

Fig. 4. The MSE of phase offset estimation (normalized by $4 \pi^{2}$ ) v.s. $E_{b} / N_{0}$. $\mathrm{NFR}=10 \mathrm{~dB} . \mathbf{A}: L=1 . \mathbf{B}: L=3$.

[8] A.-A. D'Amico, U. Mengali, and M. Morelli, "Multipath channel estimation for the uplink of a DS-CDMA system," in Proc. of IEEE Globecom, Apr. 28-May. 02, 2002. Vol.1-5, pp. 16-20.

[9] D. Angelosante, E. Grossi, G. B. Giannakis, and M. Lops, "Sparsityaware estimation of CDMA system parameters," in Eurasip Journal on Advances in Signal Processing, vol.2010, Article ID 417981, 10 pages, 2010. doi:10.1155/2010/417981.

[10] R. Tibshirani, "Regression shrinkage and selection via the Lasso," in $J$ of the Royal Stat. Society: Series B, vol. 58, no. 1, pp. 267-288, 1996.

[11] L. Hanzo, M. Münster, B. J. Choi, and T. Keller, OFDM and MC-CDMA for broadband multi-user communications, WLANs and broadcasting, John Wiley \& Sons. ISBN 0-470-85879-6, 2003.

[12] D. Divsalar, M. K. Simon, and D. Raphaeli, "Improved parallel interference cancellation for CDMA," IEEE Trans. on Comm., vol.46, no.2, pp. 258-268, Feb. 1998.

[13] S. Kondo, and L. B. Milstein, "Performance of multicarrier DS CDMA systems," in IEEE Trans. on Commun., vol.44, no.2, pp.238-246, Feb. 1996.

[14] L.-L. Yang, and L. Hanzo, "Performance of generalized multicarrier DSCDMA using various chip waveforms," in IEEE Trans. on Commun., vol.51, no.5, pp. 748-752, May 2003.

[15] D. L. Donoho, and Y. Tsaig "Fast solution of L1-norm minimization problems when the solution may be sparse," in IEEE Trans. Inform. Theory, vol. 54, no. 11, pp. 4789-4812, Nov. 2008.

[16] Y. Labit, D. Peaucelle, and D. Henrion "SeDuMi interface 1.02CA tool for solving LMI problems with SeDuMi," in Proceedings of the IEEE International Symposium on Computer, Glasgow, UK, Sept. 2002, pp. 272-277.

[17] J. Löfberg "YALMIP: A toolbox for modeling and optimization in MATLAB," in Proceedings of IEEE International Symposium on Computer Aided Control Systems Design, Sept. 2004, pp.284-289. 